\title{
Evidence-Based PET for Cardiac Diseases
}

\author{
Christel H. Kamani, Marie-Madeleine Meyer, \\ Sarah Boughdad, Nathalie Testart, \\ Marie Nicod Lalonde, Gilles Allenbach, \\ Mario Jreige, Niklaus Schaefer, Giorgio Treglia, \\ and John O. Prior
}

\subsection{Introduction}

This chapter summarizes 15 meta-analyses published in the literature on the use of PET for cardiac diseases, with the majority $(n=8)$ concerning the diagnosis of coronary artery disease (CAD) using myocardial perfusion imaging (MPI) in comparison to other modalities. Second in the number of published meta-analyses, three studies

C. H. Kamani · M.-M. Meyer · S. Boughdad N. Testart · M. Nicod Lalonde · G. Allenbach · M. Jreige Department of Nuclear Medicine and Molecular Imaging, Lausanne University Hospital, Lausanne, Switzerland

N. Schaefer · J. O. Prior $(\bowtie)$

Department of Nuclear Medicine and Molecular Imaging, Lausanne University Hospital and University of Lausanne, Lausanne, Switzerland e-mail: john.prior@chuv.ch

\section{G. Treglia}

Department of Nuclear Medicine and Molecular Imaging, Lausanne University Hospital

and University of Lausanne, Lausanne, Switzerland

Clinic of Nuclear Medicine and Molecular Imaging, Imaging Institute of Southern Switzerland,

Ente Ospedaliero Cantonale,

Bellinzona and Lugano, Switzerland

Health Technology Assessment Unit, Academic Education, Research and Innovation Area,

Ente Ospedaliero Cantonale, Bellinzona, Switzerland e-mail: giorgio.treglia@eoc.ch concentrated on the prognostic value of MPI for adverse cardiovascular events. Finally, one metaanalysis was published on the use of PET for four indications among myocardial viability assessment, the presence of microvascular disease (impaired coronary vascular function in absence of obstructive, epicardial CAD), the use of cardiac hybrid imaging and the diagnostic of cardiac amyloidosis.

\subsection{Myocardial Blood Flow Perfusion}

\subsubsection{Performance of PET/CT in the Assessment of Myocardial Perfusion in Comparison to Other Myocardial Perfusion Imaging Modalities}

Since the years 1950s, the technology of image acquisition in nuclear cardiology has progressively evolved from initial planar to more recent rapid hybrid cadmium-zinc-telluride (CZT) single-photon emission computed tomography/ computed tomography (SPECT/CT) and digitalized 3-dimensional (3D) positron emission tomography/computed tomography (PET/CT). Contemporary to this evolution, advances in the computation of acquired quantitative data using more performant software have led to a more 
objective, digital-based assessment of the pathophysiological processes underlying cardiovascular diseases, from the stenosis of epicardial coronary artery to microvascular dysfunction, including coronary vasomotor as well as endothelial dysfunction. In the meantime, new radiotracers for perfusion imaging have been developed, from potassium-43 $\left({ }^{43} \mathrm{~K}\right)$ to thallium$201\left({ }^{201} \mathrm{Tl}\right)$ and technetium-99 metastablelabelled $\left({ }^{99 \mathrm{~m}} \mathrm{Tc}\right)$ radiotracers for SPECT/CT; rubidium-82 $\left({ }^{82} \mathrm{Rb}\right)$, oxygen-15-labelled water $\left(\left[{ }^{15} \mathrm{O}\right]-\mathrm{H}_{2} \mathrm{O}\right)$, nitrogen-13-labelled ammonia $\left(\left[{ }^{13} \mathrm{~N}\right]-\mathrm{NH}_{3}\right)$, and still under development F-18 fluorine-labelled radiotracers for PET/CT.

Apart from nuclear techniques using SPECT/ $\mathrm{CT}$ and PET/CT, numerous non-invasive modalities have been developed to assess the myocardial perfusion, including dobutamine stress echocardiography (DSE), cardiac magnetic resonance (CMR), computed tomographic myocardial perfusion imaging (CT-MPI), fractional flow reserve derived from computed tomography (FFR-CT), with different diagnostic performances [1-4]. The development of these non-invasive modalities aims to contribute to counteract the burden of coronary artery disease (CAD), as the latest represents one of the leading causes of death and disability in developing countries [5]. In the field of nuclear imaging, especially PET/CT, there are accumulating evidences on the importance of flow quantification to guide management of stable CAD [6, 7], suggesting the potential role of $\mathrm{PET} / \mathrm{CT}$ as gatekeeper for invasive coronary angiography (ICA). Therefore, following this paradigm change in the treatment of patients with $\mathrm{CAD}$, it is of great importance to accurately select the right non-invasive imaging test evaluating the hemodynamic significance of a coronary stenosis for the right patient.

Jaarsma et al. [3] performed for the first time a meta-analysis on direct comparison of the diagnostic accuracy of three imaging modalities, PET/CT, CMR and SPECT/CT, for the diagnosis of significant CAD, using ICA as reference standard. In this meta-analysis, PET/CT performed better than CMR and SPECT/CT.

Dai et al. [1] have also assessed in a metaanalysis the diagnostic performance of six different cardiac imaging modalities, including PET/CT, for the evaluation of altered myocardial perfusion, using fractional flow reserve (FFR) derived from ICA as the reference standard. Among these imaging modalities, PET/CT as well as CMR and CT-MPI demonstrate high accuracy to detect hemodynamically significant $\mathrm{CAD}$, as compared with SPECT/CT, DSE and FFR-CT on a patient and coronary territory basis. From these three best performing modalities, CMR and CT-MPI perform better than PET/CT in the evaluation of perfusion deficit. However, with the advent of 3D digital PET/CT, it is expected that the improvement in the spatial resolution would add an incremental value in the evaluation of perfusion deficit [8]. One of the strengths of PET/CT and CMR over other perfusion imaging modalities is the ability to absolutely quantify myocardial blood flow, as this has been well demonstrated to be a strong predictor of coronary artery disease [9]. In this field, PET/CT performs better than CMR according to these data. Using PET/CT, different parameters are generated for the quantitative assessment of myocardial blood flow: hyperemic MBF (hMBF) defined as the myocardial blood flow during hyperemic stress test; resting MBF (rMBF) defined as the myocardial blood flow in resting conditions; relative myocardial blood flow (RBF) defined as the ratio from hMBF in the stenosed coronary territory to hMBF of a reference non-stenotic territory; and myocardial flow reserve (MFR) defined as the ratio from $\mathrm{hMBF}$ and rMBF [10]. Evidences have demonstrated a discrepancy in the diagnostic accuracy of different MBF parameters [1, 10]. Indeed, hMBF has been found to be more accurate than CFR and FFR in the detection of significant coronary artery disease using either FFR derived from ICA or visual evaluation of the severity of the stenosis during ICA, as well as both as reference standard. The limited performance of CFR regarding sensibility and specificity as compared to the two other parameters is linked to the clinically relevant coronary pathophysiology, as MFR evaluates the global vascular response to hyperemia [9]. Thus, epicardial coronary stenosis (from mild diffuse to focal severe) and microvascular dysfunction (from vasomotor tone to endothelial 
function) are determinants for the assessment of MFR. Alterations of one of these parameters in resting conditions can already lead to an activation of compensatory vasodilation of the resistance vessels in the microcirculation, resulting in an alteration of rMBF [11]. Thus, MFR could be limited in the detection of epicardial coronary stenosis in the presence of diffuse atherosclerosis. However, in contrary to hMBF, MFR has the best prediction value for major adverse cardiovascular events (MACE) when comparing both parameters [9]. FFR showed the best specificity from all three parameters. However, unlike the two other parameters, it requires a coronary territory free from relevant coronary stenosis, what can be problematic in patients with coronary 3-vessels disease. And this explains the limited sensibility of this parameter. The results of this meta-analysis are in line with the previous published meta-analysis from Takx et al. [2], with all patients presenting an intermediate epicardial coronary stenosis in ICA, further evaluated by FFR. Interestingly, in this meta-analysis, posttest probabilities following a negative respectively a positive test result in PET/CT and CMR was calculated (derived from pre-test probability and likelihood ratio), with high accuracy, ranging from $9 \%$ to $11 \%$ respectively from $84 \%$ to $85 \%$. Indeed, over sensitivity and specificity, the clinical performance of a non-invasive perfusion modality can be best assessed by the ability of post-test probability to confidently rule-in (posttest probability $\geq 85 \%$ ) or rule-out (post-test probability $\leq 15 \%$ ) relevant $\mathrm{CAD}$. In this line, a recent meta-analysis has investigated the ranges of pre-test probability of significant CAD, in which five imaging modalities, including PET/ CT, can highlight an accurate post-test probability [12]. For this purpose, anatomic and functional reference standard were used, derived from ICA and FFR respectively with cut-off values for determining significant CAD for a stenosis $>50 \%$ on ICA or a FFR $\leq 0.80$. Depending on the reference standard used, PET/CT as well as the other functional imaging techniques (SPECT/CT, CMR) performed different. Indeed, using anatomic reference standard, they all showed only moderate accuracy, whereas there was a signifi- cant improvement of their performance when using functional reference standard.

When comparing nuclear cardiology modalities together, PET/CT appears more advantageous than SPECT/CT in many aspects in two meta-analyses [13, 14]. PET/CT has a higher spatial resolution resulting from better count sensitivity and higher energy of their radiotracers. Moreover, PET/CT has a better image quality due to short half-life of their radiotracers leading to higher signal-to-noise ratio. This short half-life of the radiotracers enables lower patient radiation exposure as compared to SPECT, with up to tenth, respectively, half of a standard dose for ${ }^{201} \mathrm{Tl}$ and ${ }^{99 \mathrm{~m}} \mathrm{Tc}$ as compared to ${ }^{82} \mathrm{Rb}$. And when evaluating the efficacy of transmyocardial laser revascularization (TMR) in patients with refractory angina not amenable to conventional percutaneous or surgery revascularization, PET/CT, in contrary to SPECT/CT, demonstrates an improvement of the subendocardial to subepicardial ratio in the follow-up, where SPECT/CT showed no changes in a meta-analysis [15]. Thus, PET/CT, as compared to SPECT/CT, did have better resolution to assess the subendocardial and subepicardial perfusion, which is a relevant factor for the detection and follow-up of patients with CAD.

There are some limitations who have to be taken into consideration when interpreting the results of the most discussed meta-analysis. In the last decade, evidences from randomized controlled trials highlighted the importance of considering the hemodynamic significance of epicardial coronary lesion using fractional flow reserve (FFR) than just the traditional visual assessment of the severity of the epicardial coronary lesion in the decision of revascularization [16]. Indeed, these evidences demonstrate a discrepancy between the visual assessment of the severity and the functional significance of the lesion, with impact on the outcome when revascularizing or not. Moreover, in many patients with stable CAD and epicardial coronary stenosis, the non-inferiority of optimal medical treatment (OMT) over percutaneous coronary intervention (PCI) on top of OMT has been well demonstrated [17]. Therefore, using visual 
assessment of the severity of coronary stenosis as reference could have introduced significant bias of the present results.

FFR is a surrogate of the coronary perfusion, derived from measurement of the coronary pressure before and after the stenosis. Thereby, it would ideally represent the proportion of blood flow available to the myocardium distally from the stenosis, as compared to the one in the absence of coronary stenosis [18]. Using FFR as reference standard to evaluate the accuracy of coronary perfusion, PET/CT is largely questionable for many reasons. First, early evidences from the 1980s have demonstrated the predominant role of coronary flow over coronary perfusion pressure for the maintenance of an adequate myocardial function. In this animal experimental study, the significant reduction of coronary perfusion pressure to values equivalent to an FFR around 0.4 did not lead to alteration of the myocardial function as long as the coronary flow was maintained [19]. Second, perfusion imaging modalities were used to validate FFR [20], making it less suitable as reference standard to determine the accuracy of perfusion PET/CT. Third, recent data from large, multicenter, prospective randomized trial demonstrate that a significant proportion of patients $(60 \%$ of the study population) with epicardial stenosis showing FFR $\leq 0.8$ did not require coronary revascularization [21]. Following all these evidences, the results of these meta-analyses should be interpreted with care. It is not excluded that one of these imaging modalities is more accurate than what it was found, but it could not be demonstrated due to the use of FFR as reference standard. Other bias could also have an impact on the results of these different meta-analyses, such as the use of different PET/ CT scans and different PET/CT scan protocols, the difference in the prevalence of CAD in the different studies, the difference in the threshold of FFR and visual assessing of the severity of the coronary stenosis. Moreover, most of the metaanalyses have evaluated the imaging modalities as a stand-alone technique, without integrating other clinical parameters such as age and gender as well as other para-clinical parameters such as ventricular function, which could have increased the accuracy of the imaging modality. Finally, even if considerable efforts have been made to reduce the radiation exposure of patients undergoing a PET/CT, it remains a matter of concern when comparing to other imaging modalities such as CMR with no radiation exposure.

Despite the significant advances in the assessment of patients with $\mathrm{CAD}$, further investigations are still needed to overcome these limitations, thus bringing a new step of comprehension in the art of performing the right exam to the right patient.

\subsubsection{Prognostic Value of Myocardial PET}

PET MPI is increasingly being used for an accurate assessment of myocardial ischemia in patients with known or suspected coronary artery disease (CAD). It has been proposed by the American Heart Association that PET MPI may play a role as a novel cardiovascular biomarker, allowing better risk stratification of patients with CAD.

A first meta-analysis by Siontis et al. [22] specifically evaluated the incremental prognostic value that PET MPI added to standard risk factors in patients with known or suspected CAD. They selected 20 studies (with possible overlap in 5) totaling 22,203 patients, during a long span of 20 years, with only seven prospective studies. There was considerable heterogeneity among studies as MPI acquisition protocols, image analysis and selected radiotracer were not standardized among the studies. Only five studies reported changes in model classification, discrimination and risk stratification. PET MPI improved risk classification in four out of these five studies Despite the limitations of the metaanalysis, the authors were able to show that there is a strong association between abnormal perfusion (both by quantitative and qualitative analysis) and patient's outcome. They concluded that the limited data suggest that PET MPI may improve risk stratification, but this should be confirmed by data from larger and standardized prospective randomized controlled trials. 
The second systematic review and metaanalysis by Chen et al. [23] focused on evaluating the prognostic value of normal PET MPI in patients with suspected or known CAD. They selected 11 studies with a total of 20,471 patients in whom PET MPI was performed for the diagnosis of coronary artery disease and with evaluation of cardiovascular death, all-cause death and major cardiovascular events (MACE) at a followup $\geq 3$ months. They found highly significant negative predictive values for cardiac death (98.8\%; 95\% confidence interval (CI), 97.64$99.39 \%)$, all-cause death $(94.8 \%$; $95 \%$ CI: 92.99-96.30\%) and MACE (90.2\%; 95\% CI: 78.01-96.03\%), with a reasonable follow-up period of 36.9 months, 26.8 months and 35.7 months, respectively. In a subgroup analysis, there was no significant difference in negative predictive value in studies with a normal PET MPI determined by absence of perfusion abnormalities compared to those using coronary flow reserve. An important limitation is that the authors were not able to differentiate patients with known coronary artery disease from patients with only clinical suspicion.

The third wide meta-analysis carried by Smulders et al. [24] compared several noninvasive tests for depiction of coronary artery disease. The aim was to evaluate the prognostic value of negative non-invasive cardiac investigations in patients with suspected or known CAD. They compared CT angiography, stress echocardiography, cardiac magnetic resonance, exercise electrocardiographic testing, SPECT and PET. The evaluated outcome was annual event rate for cardiac death and myocardial infarction. They included 165 studies with a total of 122,721 patients. Annual event rates (AER) after a negative test varied among the imaging modalities (between 0.32 for $\mathrm{CT}$ angiography and 1.36 for stress echocardiography). Some modalities are preferred in populations at higher event risk or known CAD. The authors supposed the different patient population among studies could explain the differences between modalities. Indeed, when adjusting for the population risk, the AER was similar between modalities. This meta-analysis supports that a negative non-invasive cardiac study provides predictive information with good accuracy, which should influence the clinical practice by reducing the need of further cardiac investigations.

In the last years, there is growing scientific evidence describing the value of non-invasive cardiac investigations, not only as a diagnostic tool but also as a predictive prognosis biomarker. Specifically, a negative test has an excellent prognosis, which should reasonably reassure the patient.

\subsection{Assessment of Myocardial Viability}

The concept of hibernating myocardium has been adopted in the early 1980 s following the cumulating evidences of an improvement of the myocardial regional contractile function after an aorto-coronary bypass surgery [25]. It described a condition of altered left ventricular contractile function as a consequence of prolonged reduced coronary blood supply at rest, with improving potential following coronary revascularization. Different non-invasive imaging modalities, such as dobutamine stress echocardiography, cardiac magnetic resonance imaging and nuclear imaging, focusing on different aspects of the viable myocardium, have been proposed to identify viability in dysfunctional myocardial segments, with different diagnostic accuracy [26]. Among all these modalities, PET is considered as the gold standard [27].

In the meta-analysis of Tsai et al. [28], the authors investigated the accuracy of SPECT for the diagnosis of myocardial viability in patients with CAD and left ventricular dysfunction as compared to ${ }^{18} \mathrm{~F}$-FDG PET. Although this metaanalysis was not centred on PET, which was only used as the gold-standard comparator for SPECT, it is the only one published about viability involving PET/CT. The authors found eight prospective studies totaling 320 patients (3580 segments analysed). The criteria used for SPECT viability were diverse in these eight studies, but most frequently involved the presence of myocardial rest perfusion visible (e.g. $>50 \%$ or $>55 \%$ ), while 
two studies also involved ${ }^{18} \mathrm{~F}-\mathrm{FDG}$ in the criteria for SPECT viability (usually with SPECT vs. PET mismatch indicating viability).

SPECT as compared to PET resulted in the following pooled values: pooled sensitivity $82 \%$ (95\% CI $81-84 \%$ ), pooled specificity $88 \%$ (95\% CI 86-90\%), diagnostic odd ratio 62.6 (95\% CI 19.3-203) and the area under the receiving operating characteristic curve was $0.945 \pm 0.026$. There was a significant heterogeneity among studies especially in the definition of myocardial viability and the largest study included 58 patients, but the QUADAS quality assessment was of excellent quality and the funnel plot indicated no publication bias.

Thus, this meta-analysis showed that SPECT can be used adequately to assess myocardial viability in patients with CAD and left ventricular dysfunction. However, nowadays SPECT appears to be an outdated modality for assessing myocardial viability, and cardiac PET would be preferred for this diagnosis.

\subsection{Microvascular Disease}

Coronary artery disease (CAD) has often been exclusively associated to epicardial coronary abnormalities, as visualized during invasive coronary angiography (ICA). Nowadays, there are more evidences on the involvement of the entire coronary circulation, including the microcirculation, in the development of symptoms related to CAD [29]. This issue has been highlighted in a large cohort of symptomatic patients undergoing a diagnostic ICA for suspected obstructive epicardial CAD, where about $60 \%$ of the patients did not have a significant obstructive CAD (stenosis $\leq 50 \%$ ) [30]. Microvascular dysfunction may encounter for a significant number of symptomatic patients without relevant obstructive CAD [29, 31]. Coronary microvascular disease (CMD) englobes a group of disorders affecting the structure and function of the coronary microcirculation in relation with endothelial dysfunction or a dysregulation of the vascular smooth muscle. Numerous non-invasive techniques have been proposed to assess the coronary microvascular function, such as PET/CT, dynamic CT-MPI and CMR [32]. Among these, PET/CT is the most validated modality with good reproducibility and accuracy.

In a systematic review and meta-analysis, Brainin et al. investigated the consequences of impaired coronary vascular function in the absence of obstructive coronary arterial disease [33]. In six studies ( $n=1192$ patients) endothelial dependent epicardial dysfunction was assessed, five of them measured coronary flow reserve (CFR) during angiography using coldpressure test or acetylcholine stimulation. In one study, CFR was measured using cold-pressure testing during PET. In a pooled analysis, the relative risk (RR) of 2.49 for cardiovascular events was significantly increased. In another group of ten studies $(n=5134)$, non-endothelial dependent epicardial dysfunction was assessed measuring coronary flow velocity reserve using echocardiography. These studies showed an increased RR of 4.58. In the last group including ten studies ( $n=3687$ patients) measuring CFR by PET, non-endothelial dysfunction was associated with an increased RR of 2.44 .

These results underline that coronary vascular dysfunction as assessed by PET/CT has a prognostic value on cardiovascular events in a group of patients without obstructive coronary arterial disease. RR remained significantly elevated when excluding patients with diabetes or angina, as it remained elevated when including only patients with one specific stressor. Even if the number of studies remained small with different methods applied, impaired coronary vascular function in the absence of obstructive coronary disease should be reported for its prognostic value and considered as a potential therapeutic target.

\subsection{Cardiac Hybrid Imaging}

Cardiac multimodality (hybrid) imaging is a technique relying on the combination (on a sideby-side or fusion mode) of imaging modalities providing in one hand cardiac morphological data, such as CT, echocardiography or CMR, and in the other hand imaging modalities providing myocardial functional data, such as PET/CT. The goal of this combination is to gain information of 
these different imaging modalities in a complementary fashion in order to better guide coronary revascularization [34].

Coronary CT angiography (CCTA) has high negative predictive value and sensitivity for diagnosing obstructive $\mathrm{CAD}$; however, its positive predictive value and specificity are lower. In particular, CCTA may overestimate coronary artery stenosis. Therefore, a hybrid approach combining CCTA with MPI modalities_-including PET, SPECT and CMR - may help diminish the falsepositive rate of CCTA by fusing anatomic data derived from CCTA with functional data obtained through MPI, thus potentially overcoming CCTA limitations. To evaluate the clinical utility of this approach, Rezvi et al. [35] conducted a systematic literature review and meta-analysis comparing the diagnostic performance of hybrid cardiac imaging modalities with stand-alone CCTA for assessing obstructive $\mathrm{CAD}$, using as a reference standard invasive coronary angiography (ICA).

The results of the meta-analysis showed that, at the per-patient level, CCTA and MPI demonstrated comparable sensitivity $(p=0.35)$, but CCTA displayed lower specificity (66\%) compared to MPI (83\%) for predicting obstructive CAD; at the per-vessel level, specificity of CCTA and MPI was similar $(p=0.02)$, and sensitivity was higher for CCTA (89\%) than for MPI alone $(78 \%)(p<0.001)$.

When the diagnostic performance of hybrid versus CCTA imaging method was examined on a per-patient basis, sensitivity, negative likelihood ratio (LR-) and diagnostic odds ratio (DOR) of hybrid imaging techniques compared to CCTA to detect obstructive CAD were, respectively, 91\%, 0.11 and 159.00, versus 90\%, 0.06 and $53.80(p>0.05$, for all). Specificity and positive likelihood ratio $(\mathrm{LR}+)$ were higher for hybrid imaging compared to stand-alone CCTA (93\% and 12.80 versus $66 \%$ and 3.39 , respectively). At the per-vessel level, summary receiver-operator curves (sROC) demonstrated a statistically significant and higher area under the curve (AUC) value for hybrid imaging than for CCTA (0.97 versus 0.93 , respectively; $p=0.047$ ).

In conclusion, hybrid imaging techniques outperformed stand-alone CCTA for detecting obstructive $\mathrm{CAD}$ in patients undergoing both anatomic and functional testing, demonstrating higher specificity and LR+ on a per-patient basis. In addition, at the per-vessel level, hybrid imaging could better identify CAD based on sROC curves; however, sensitivity was comparable for hybrid versus stand-alone CCTA imaging.

\subsection{Cardiac Amyloidosis}

Amyloidosis encompasses a group of infiltrative disorders characterized by extracellular accumulation of fibrillary proteins, leading to functional impairment of different tissues and organs, including the heart. In patients with systemic amyloidosis, cardiac involvement could lead to significantly increased morbidity and mortality. The most common types of cardiac amyloidosis are systemic light chain (AL) and transthyretin (ATTR) amyloidosis. This differentiation is of importance as it differs in treatment and prognosis, untreated patients with AL amyloidosis having the poorest prognosis [36]. ATTR has been long time considered as a rare disease because of the lack of disease awareness, its quiescent nature at the beginning and the heterogeneity of symptoms when clinical manifest. Nowadays, accumulating evidences showed that it may be more prevalent than thought, especially in certain groups of patients such as older people or patients with aortic stenosis [37]. Moreover, with the emergence of new treatment options, early diagnosis is critical because of the better effectiveness of the treatment in the earlier course of the disease [38]. In line with this, bone scintigraphy as a non-invasive tool in the flowchart to accurately differentiate between AL and ATTR amyloidosis is well established [39]. Moreover, recent development in amyloid tracers for positron emission tomography (PET) could lead to an early diagnosis and potentially improve the prognosis of those patients. In that setting, we shortly present the results of the first systematic review and meta-analysis on the diagnostic performance of amyloid PET imaging in cardiac amyloidosis by Kim et al. [40]. The authors also reported the potential additional value of using semi-quantitative parameters for the diagnosis of cardiac 
amyloidosis and for distinguishing between AL and ATTR amyloidosis.

Six retrospective studies with a total of 98 patients (69 patients with systemic amyloidosis and 29 control patients) were included in this meta-analysis. Among amyloid radiotracers, ${ }^{11} \mathrm{C}$ PIB was the most commonly used in four studies, whereas ${ }^{18} \mathrm{~F}$-Florbetapir and ${ }^{18} \mathrm{~F}$-Florbetaben were each used in one study.

In the whole cohort (six studies), the pooled sensitivity was 0.95 (95\% CI 0.87-0.99), and pooled specificity was 0.98 (95\% CI $0.87-1.00)$. Positive likelihood ratio (LR) was 10.130 (95\% CI 3.749-27.376), negative LR was 0.100 (95\% CI 0.045-0.221), and diagnostic odds ratio was 148.83 (95\% CI = 34.026-650.98). Summary receiver operating characteristic curve showed high performance with an area under curve of 0.9731 with a standard error of 0.0156 . Looking only at the four ${ }^{11} \mathrm{C}$-PIB studies, similar results were found with a diagnostic odds ratio of 134.19 (95\% CI 24.039-749.03).

The use of semi-quantitative parameters was reported in four studies (three with ${ }^{11} \mathrm{C}$-PIB and one with ${ }^{18} \mathrm{~F}$-Florbetaben) and consisted of retention index (RI) and/or target-to-background ratio (TBR). RI derived from dynamic acquisitions (RI = mean myocardial SUV/integral of the arterial time-activity curve). By contrast, TBR was extracted from static acquisitions $(\mathrm{TBR}=\max$ or mean myocardial SUV/mean SUV of the descending thoracic aorta). Patients with cardiac amyloidosis had significantly higher RI and TBR values in comparison to control patients. Using both, pooled standardized mean difference (SMD) was of $1.42 \quad(95 \%$ CI $0.83-2.01$; $p<0.001)$. The performance of RI and TBR for discriminating between AL and ATTR amyloidosis was assessed in three studies (two with ${ }^{11} \mathrm{C}$ PIB and one with ${ }^{18} \mathrm{~F}$-Florbetaben) and showed significantly higher uptake in the former (pooled SMD $=0.96 ; 95 \%$ CI 0.13-1.79; $p<0.001)$.

To resume, amyloid PET imaging presents with strong performance to diagnose cardiac amyloidosis with high sensitivity and specificity $(\geq 95 \%)$. The addition of semi-quantitative parameters, such as RI and TBR, could help improve the diagnosis and accurately differentiate between AL and ATTR amyloidosis.

\section{References}

1. Dai N, Zhang X, Zhang Y, Hou L, Li W, Fan B, et al. Enhanced diagnostic utility achieved by myocardial blood analysis: a meta-analysis of noninvasive cardiac imaging in the detection of functional coronary artery disease. Int J Cardiol. 2016;221:665-73. https://doi. org/10.1016/j.ijcard.2016.07.031.

2. Takx RA, Blomberg BA, El Aidi H, Habets J, de Jong PA, Nagel E, et al. Diagnostic accuracy of stress myocardial perfusion imaging compared to invasive coronary angiography with fractional flow reserve meta-analysis. Circ Cardiovasc Imaging. 2015;8(1). https://doi.org/10.1161/CIRCIMAGING.114.002666.

3. Jaarsma C, Leiner T, Bekkers SC, Crijns HJ, Wildberger JE, Nagel E, et al. Diagnostic performance of noninvasive myocardial perfusion imaging using single-photon emission computed tomography, cardiac magnetic resonance, and positron emission tomography imaging for the detection of obstructive coronary artery disease: a meta-analysis. J Am Coll Cardiol. 2012;59(19):1719-28. https://doi.org/ 10.1016/j.jacc.2011.12.040.

4. Yang K, Yu SQ, Lu MJ, Zhao SH. Comparison of diagnostic accuracy of stress myocardial perfusion imaging for detecting hemodynamically significant coronary artery disease between cardiac magnetic resonance and nuclear medical imaging: a metaanalysis. Int J Cardiol. 2019;293:278-85. https://doi. org/10.1016/j.ijcard.2019.06.054.

5. Benjamin EJ, Muntner P, Alonso A, Bittencourt MS, Callaway CW, Carson AP, et al. Heart disease and stroke statistics-2019 update: a report from the American Heart Association. Circulation. 2019;139(10):e56-e528. https://doi.org/10.1161/ CIR.0000000000000659.

6. Bober RM, Milani RV, Oktay AA, Javed F, Polin NM, Morin DP. The impact of revascularization on myocardial blood flow as assessed by positron emission tomography. Eur J Nucl Med Mol Imaging. 2019;46(6):1226-39. https://doi.org/10.1007/s00259019-04278-8.

7. Gould KL, Johnson NP, Roby AE, Nguyen T, Kirkeeide R, Haynie M, et al. Regional, artery-specific thresholds of quantitative myocardial perfusion by PET associated with reduced myocardial infarction and death after revascularization in stable coronary artery disease. J Nucl Med. 2019;60(3):410-7. https:// doi.org/10.2967/jnumed.118.211953.

8. Bendriem B, Reed J, McCullough K, Khan MR, Smith AM, Thomas D, et al. The continual innovation of commercial PET/CT solutions in nuclear cardiology: Siemens Healthineers. J Nucl Cardiol. 2018;25(4):1400-11. https://doi.org/10.1007/s12350018-1262-3.

9. Gupta A, Taqueti VR, van de Hoef TP, Bajaj NS, Bravo PE, Murthy VL, et al. Integrated noninvasive physiological assessment of coronary circulatory function and impact on cardiovascular mortality in patients with stable coronary artery disease. Circulation. 
2017;136(24):2325-36. https://doi.org/10.1161/ CIRCULATIONAHA.117.029992.

10. Cho SG, Lee SJ, Na MH, Choi YY, Bom HH. Comparison of diagnostic accuracy of PETderived myocardial blood flow parameters: a meta-analysis. J Nucl Cardiol. 2018. https://doi. org/10.1007/s12350-018-01476-z.

11. Johnson NP, Gould KL. Integrating noninvasive absolute flow, coronary flow reserve, and ischemic thresholds into a comprehensive map of physiological severity. JACC Cardiovasc Imaging. 2012;5(4):430 40. https://doi.org/10.1016/j.jcmg.2011.12.014.

12. Knuuti J, Ballo H, Juarez-Orozco LE, Saraste A, Kolh P, Rutjes AWS, et al. The performance of noninvasive tests to rule-in and rule-out significant coronary artery stenosis in patients with stable angina: a meta-analysis focused on post-test disease probability. Eur Heart J. 2018;39(35):3322-30. https://doi. org/10.1093/eurheartj/ehy267.

13. Mc Ardle BA, Dowsley TF, deKemp RA, Wells GA, Beanlands RS. Does rubidium-82 PET have superior accuracy to SPECT perfusion imaging for the diagnosis of obstructive coronary disease?: a systematic review and meta-analysis. J Am Coll Cardiol. 2012;60(18):1828-37. https://doi. org/10.1016/j.jacc.2012.07.038.

14. Parker MW, Iskandar A, Limone B, Perugini A, Kim $\mathrm{H}$, Jones $\mathrm{C}$, et al. Diagnostic accuracy of cardiac positron emission tomography versus single photon emission computed tomography for coronary artery disease: a bivariate meta-analysis. Circ Cardiovasc Imaging. 2012;5(6):700-7. https://doi.org/10.1161/ CIRCIMAGING.112.978270.

15. Iwanski J, Knapp SM, Avery R, Oliva I, Wong RK, Runyan RB, et al. Clinical outcomes meta-analysis: measuring subendocardial perfusion and efficacy of transmyocardial laser revascularization with nuclear imaging. J Cardiothorac Surg. 2017;12(1):37. https:// doi.org/10.1186/s13019-017-0602-8.

16. Bech GJ, De Bruyne B, Pijls NH, de Muinck ED, Hoorntje JC, Escaned J, et al. Fractional flow reserve to determine the appropriateness of angioplasty in moderate coronary stenosis: a randomized trial. Circulation. 2001;103(24):2928-34. https://doi.org/ 10.1161/01.cir.103.24.2928.

17. Shaw LJ, Berman DS, Maron DJ, Mancini GB, Hayes SW, Hartigan PM, et al. Optimal medical therapy with or without percutaneous coronary intervention to reduce ischemic burden: results from the Clinical Outcomes Utilizing Revascularization and Aggressive Drug Evaluation (COURAGE) trial nuclear substudy. Circulation. 2008;117(10):1283-91. https://doi. org/10.1161/CIRCULATIONAHA.107.743963.

18. Pijls NH, van Son JA, Kirkeeide RL, De Bruyne B, Gould KL. Experimental basis of determining maximum coronary, myocardial, and collateral blood flow by pressure measurements for assessing functional stenosis severity before and after percutaneous transluminal coronary angioplasty. Circulation. 1993;87(4):1354-67. https://doi.org/10.1161/01. cir.87.4.1354.
19. Smalling RW, Kelley K, Kirkeeide RL, Fisher DJ. Regional myocardial function is not affected by severe coronary depressurization provided coronary blood flow is maintained. J Am Coll Cardiol. 1985;5(4):948-55. https://doi.org/10.1016/ s0735-1097(85)80438-1.

20. Pijls NH, De Bruyne B, Peels K, Van Der Voort $\mathrm{PH}$, Bonnier HJ, Bartunek JKJJ, et al. Measurement of fractional flow reserve to assess the functional severity of coronary-artery stenoses. N Engl J Med. 1996;334(26):1703-8. https://doi.org/10.1056/ NEJM199606273342604.

21. De Bruyne B, Pijls NH, Kalesan B, Barbato E, Tonino PA, Piroth Z, et al. Fractional flow reserve-guided PCI versus medical therapy in stable coronary disease. $\mathrm{N}$ Engl J Med. 2012;367(11):991-1001. https://doi. org/10.1056/NEJMoa1205361.

22. Siontis KC, Chareonthaitawee P. Prognostic value of positron emission tomography myocardial perfusion imaging beyond traditional cardiovascular risk factors: systematic review and meta-analysis. Int J Cardiol Heart Vasc. 2015;6:54-9. https://doi. org/10.1016/j.ijcha.2015.01.005.

23. Chen A, Wang H, Fan B, Xu Y, Chen W, Dai N. Prognostic value of normal positron emission tomography myocardial perfusion imaging in patients with known or suspected coronary artery disease: a meta-analysis. Br J Radiol. 2017;90(1074):20160702. https://doi.org/10.1259/bjr.20160702.

24. Smulders MW, Jaarsma C, Nelemans PJ, Bekkers S, Bucerius J, Leiner T, et al. Comparison of the prognostic value of negative non-invasive cardiac investigations in patients with suspected or known coronary artery disease-a meta-analysis. Eur Heart J Cardiovasc Imaging. 2017;18(9):980-7. https://doi.org/10.1093/ ehjci/jex014.

25. Rahimtoola SH. The hibernating myocardium. Am Heart J. 1989;117(1):211-21. https://doi. org/10.1016/0002-8703(89)90685-6.

26. Schinkel AF, Bax JJ, Poldermans D, Elhendy A, Ferrari R, Rahimtoola SH. Hibernating myocardium: diagnosis and patient outcomes. Curr Probl Cardiol. 2007;32(7):375-410. https://doi. org/10.1016/j.cpcardiol.2007.04.001.

27. Nekolla SG, Martinez-Moeller A, Saraste A. PET and MRI in cardiac imaging: from validation studies to integrated applications. Eur J Nucl Med Mol Imaging. 2009;36(Suppl 1):S121-30. https://doi.org/10.1007/ s00259-008-0980-1.

28. Tsai JP, Yun CH, Wu TH, Yen $\mathrm{CH}$, Hou CJ, Kuo JY, et al. A meta-analysis comparing SPECT with PET for the assessment of myocardial viability in patients with coronary artery disease. Nucl Med Commun. 2014;35(9):947-54. https://doi. org/10.1097/MNM.0000000000000140.

29. Camici PG, Crea F. Coronary microvascular dysfunction. N Engl J Med. 2007;356(8):830-40. https://doi. org/10.1056/NEJMra061889.

30. Patel MR, Peterson ED, Dai D, Brennan JM, Redberg RF, Anderson HV, et al. Low diagnostic yield of elective coronary angiography. N Engl J 
Med. 2010;362(10):886-95. https://doi.org/10.1056/ NEJMoa0907272.

31. Ong P, Camici PG, Beltrame JF, Crea F, Shimokawa $\mathrm{H}$, Sechtem U, et al. International standardization of diagnostic criteria for microvascular angina. Int $\mathbf{J}$ Cardiol. 2018;250:16-20. https://doi.org/10.1016/j. ijcard.2017.08.068.

32. Feher A, Sinusas AJ. Quantitative assessment of coronary microvascular function: dynamic singlephoton emission computed tomography, positron emission tomography, ultrasound, computed tomography, and magnetic resonance imaging. Circ Cardiovasc Imaging. 2017;10(8). https://doi. org/10.1161/CIRCIMAGING.117.006427.

33. Brainin P, Frestad D, Prescott E. The prognostic value of coronary endothelial and microvascular dysfunction in subjects with normal or non-obstructive coronary artery disease: a systematic review and meta-analysis. Int J Cardiol. 2018;254:1-9. https:// doi.org/10.1016/j.ijcard.2017.10.052.

34. Prior JO, Farhad H, Muller O. Multimodality imaging in ischemic cardiomyopathy. Curr Cardiovasc Imaging Rep. 2014;7:9285. https://doi.org/10.1007/ s12410-014-9285-x.

35. Rizvi A, Han D, Danad I, Hartaigh BO, Lee JH, Gransar H, et al. Diagnostic performance of hybrid cardiac imaging methods for assessment of obstructive coronary artery disease compared with stand-alone coronary computed tomography angiography: a meta- analysis. JACC Cardiovasc Imaging. 2018;11(4):589_ 99. https://doi.org/10.1016/j.jcmg.2017.05.020.

36. Xin Y, Hu W, Chen X, Hu J, Sun Y, Zhao Y. Prognostic impact of light-chain and transthyretin-related categories in cardiac amyloidosis: a systematic review and meta-analysis. Hell J Cardiol. 2019;60(6):375-83. https://doi.org/10.1016/j.hjc.2019.01.015.

37. Maurer MS, Bokhari S, Damy T, Dorbala S, Drachman BM, Fontana M, et al. Expert consensus recommendations for the suspicion and diagnosis of transthyretin cardiac amyloidosis. Circ Heart Fail. 2019;12(9):e006075. https://doi.org/10.1161/ CIRCHEARTFAILURE.119.006075.

38. Maurer MS, Schwartz JH, Gundapaneni B, Elliott PM, Merlini G, Waddington-Cruz M, et al. Tafamidis treatment for patients with transthyretin amyloid cardiomyopathy. N Engl J Med. 2018;379(11):1007-16. https://doi.org/10.1056/NEJMoa1805689.

39. Gillmore JD, Maurer MS, Falk RH, Merlini G, Damy T, Dispenzieri A, et al. Nonbiopsy diagnosis of cardiac transthyretin amyloidosis. Circulation. 2016;133(24):2404-12. https://doi.org/10.1161/ CIRCULATIONAHA.116.021612.

40. Kim YJ, Ha S, Kim YI. Cardiac amyloidosis imaging with amyloid positron emission tomography: a systematic review and meta-analysis. J Nucl Cardiol. 2018;27(1):123-32. https://doi.org/10.1007/ s12350-018-1365-X.

Open Access This chapter is licensed under the terms of the Creative Commons Attribution 4.0 International License (http://creativecommons.org/licenses/by/4.0/), which permits use, sharing, adaptation, distribution and reproduction in any medium or format, as long as you give appropriate credit to the original author(s) and the source, provide a link to the Creative Commons license and indicate if changes were made.

The images or other third party material in this chapter are included in the chapter's Creative Commons license, unless indicated otherwise in a credit line to the material. If material is not included in the chapter's Creative Commons license and your intended use is not permitted by statutory regulation or exceeds the permitted use, you will need to obtain permission directly from the copyright holder. 